\title{
Effect of Community Dialogue on Self-Initiated Voluntary Medical Male Circumcision (VMMC) and Uptake of HIV Voluntary Counseling and Testing (VCT) among Male Residents of Homa-Bay County, Kenya
}

\author{
Article by Albert Ngetich ${ }^{1}$, Benard O. Abongo ${ }^{2}$, Gordon Okomo ${ }^{3}$ and Collins Ouma ${ }^{4}$ \\ ${ }^{1}$ School of Public Health, Texila American University, Nicaragua \\ ${ }^{2}$ Faculty of Science, National University of Lesotho, Lesotho \\ ${ }^{3}$ Directorate of Health Services at the Ministry of Health, Homa-Bay County, Kenya \\ ${ }^{4}$ School of Public Health and Community Development, Maseno University, Kenya \\ E-mail:bo.abongo@nul.ls ${ }^{2}$,okomogordon@hotmail.com ${ }^{3}$, collinouma@yahoo.com ${ }^{4}$
}

\begin{abstract}
Background: Voluntary medical male circumcision (VMMC) reduces transmission of HIV by about $60 \%$. The WHO and UNAIDS recommended it as a new HIV prevention intervention. Counties in western Kenya region which are predominantly occupied by a traditionally non-circumcising Luo ethnic community have low uptake of VMMC. Homa-Bay County leads in HIV and AIDS scourge, thus requires an intervention.

Methodology: Community dialogue, an interactive participatory communication has not been applied to support VMMC in Homa-Bay County. In a longitudinal study, the effect of community dialogue on self-initiated VMMC and uptake of HIV voluntary counseling and testing (VCT) was determined in male residents (aged 18-49 years) resident in Homa-Bay County, Kenya. Baseline (before intervention) and endline (after intervention) information were collected to capture the VMMC and VCT status. Total number counts accessing VCT and VMMC services were used to assess the effect of dialogue on the uptake of VMMC and VCT services.

Results: Following community dialogue, males accessing VMMC services was generally increased: from 829 (January, 2018), 1695 (February, 2018), 815 (March, 2018) and finally 3600 (April, 2018) while for VCT services: from 360 (January, 2018), 1281 (February, 2018), 615 (March, 2018) and finally 1729 (April, 2018). For both VMMC and VCT, there was a positive increment on the numbers of males accessing the services following intervention.

Conclusion: Since community dialogue is an effective intervention and increases the number of males in accessing VMMC and VCT services, it can be used as an intervention in the prevention of HIV infection.
\end{abstract}

Keywords: VMMC, VCT, community acceptability, HIV and AIDS.

\section{Introduction}

Kenya is one of the six HIV 'high burden' countries in Africa - about 1.6 million people were living with HIV infection at the end of 2013 with a possibility of increased prevalence over the next couple of years. Heterosexual transmission accounts for the majority of new infections in Kenya, just like in the rest of the world. Although the progress towards elimination of the disease is encouraging, reducing the number of new infections - estimated at 117,000 per year (UNAIDS, 2016)-remains a public health priority in the country. Given the dwindling success of individual-oriented HIV prevention interventions (Coates et al., 2008), continuing efforts tend to emphasize comprehensive interventions to better address structural and normative factors that underlie HIV transmission. In a previously developed theory, (Murdock, 1956), Murdock defined culture as a system of habits that are learned collectively and passed from one generation to the next. These habits - or social normsendure because of social pressure to conform and may prevent individuals from expressing divergent opinions or actions. According to Noelle-Neumann (Noelle-Neumann, 1974, 2014), willingness to share an opinion depends on the perception of public opinion on the subject (Scheufele and Moy, 2000). When what is perceived as the minority opinion is self-censored to avoid social isolation, a "spiral" of silence develops that prevents deviant voices from being heard (Noelle-Neumann, 1974, 
2014). This raises the question on how new social norm can emerge. The theory of bounded normative influence posits that a minority position can become the social norm by a process of normative influence through communication networks (Kincaid, 2004). These networks of people linked through information and uniform, although minority, opinions can maintain a majority status within a portion of the larger network and expand by gaining converts, thus eventually establishing its position as the norm for the larger network.

Convergence theory presents communication as a process of sharing or creating information by participants who seek to clarify information, knowledge, messages, symbols or meaning (Kincaid, 1987). Dialogue is central to the process of convergence. According to Brazilian educator Paulo Freire (2001), face-to-face dialogue that provides opportunities for critical thinking, questioning of assumptions, and the development of a new view of the world leads to conscientização (critical consciousness) among groups of participants. "Community Dialogues" was originally designed to address underlying social determinants, especially inequitable gender norms, as a fundamental step in reducing HIV risk behaviors, increasing self-efficacy to talk about and address HIV, and decreasing HIV stigma. The program has been successfully implemented elsewhere such as in the provinces of Sofala and Zambezia in Zimbabwe whereby community-based organizations (CBOs) and nongovernmental organizations (NGOs) carried out 1,134 community discussions/debates in 267 villages, reaching a total of 32,679 men and women participants during 18 months between 2009 and 2010 (Underwood et al., 2011).

In Kenya, community dialogue has been applied in several other areas such as in task shifting among community health workers in nomadic communities (Ochieng et al., 2014), in shifting social norms and enabling family planning initiatives (Wegs et al., 2016) and in identifying what motivates maternal and child nutrition peer educators based on fathers' and grandmothers' experiences in western Kenya (Martin et al., 2015). Given that the VMMC program has proven successful in reducing the HIV pandemic, and the fact that community dialogue has proven as a means to address underlying social determinants and that it can be used as a strategy to penetrate through the Luo noncircumcision cultural system, the current study established the effect of community dialogue on selfinitiated voluntary medical male circumcision (VMMC) among male residents of Homa-Bay County in Kenya.

It is well known currently that the voluntary HIV testing and counseling (VCT) is the primary entry point to HIV prevention, care and treatment services (Wachira et al., 2012). Knowing one's HIV status provides those who are negative with the opportunity to remain HIV-free and those who are HIV positive, critical links to treatment, care, support and prevention interventions to reduce reinfection and transmission of HIV to others. Although the testing coverage has improved over the years (KAIS, 2012; KDHS, 2008-2009), Kenya has not attained universal access to HIV testing. A major reason that hinders individuals from accessing HIV testing and counseling services in Kenya is low HIV risk perception (KAIS, 2007). Low HIV counselling and testing coverage in Kenya has been associated with stigma (Gilbert and Walker, 2010; Sanjobo et al., 2008), lack of transport to testing sites and lack of time for testing and counseling (Exner et al., 2002; Govindasamy et al., 2012). As a way to mitigate the challenges and barriers faced by clients in accessing the VCT, a community dialogue approach can be adopted. As attempts are being made to incorporate VCT as part of homebased testing and counseling (HBTC), which in essence is a community service, its acceptance will be influenced by the community's views about it and therefore use of sound community participation principles should be key. In an attempt to incorporate the community dialogue and access to VCT services, the current study determined the effect of community dialogue on the uptake of HIV voluntary counseling and testing (VCT) among male residents of Homa-Bay County, Kenya.

\section{Materials and methods}

\section{Study population and enrolment}

The study was carried out in Homa-Bay County within the Nyanza region, Kenya. A longitudinal study design was adopted for the study whereby uncircumcised males of Luo ethnic group aged 18-49 years and resident in Homa-Bay County were recruited into the study. Baseline information was initially collected to capture the acceptance of VMMC and factors affecting acceptability of VMMC 
and access to VCT; circumcision status and testing for HIV (reported in Ngetich et al., 2018). Only those who had a negative attitude to VMMC; not circumcised and have never undertaken HIV test were recruited into the study and a questionnaire used to collect data from them (see Appendix I). Scientific and ethical approval was sought from Maseno University's Ethics Review Committee (MUERC). Additional approval was sought from the Homa-Bay County, Director of Health. Informed consent was obtained from study participants prior to inclusion in the study. Participation into the study was on voluntary basis. There were no foreseeable risks attached to this study and information generated out of the study was treated as confidential and were stored in password-protected computers that were only accessible by the principal investigator. Feedback on the outcome of the study was shared during community feedback sessions. Participants were allowed to withdraw from the study should they wish to do so and information gathered from such withdrawing participants were not included in the final data analysis.

\section{Sampling technique}

Using a Community Health Worker-generated household register, a systematic random sampling of households was done at a sampling interval of 3 (derived from total households/targeted households i.e. $1250 / 405=3.0$ ). To be considered during the systematic random sampling of households within the villages making up Homa-Bay County, male residents had to be aged 18-49 years. Community dialogue sessions were done by recruits drawn from every village and who were familiar with the terrain and constitution of the households.

\section{Data collection}

Prior to the use of data collection tools, the tools were piloted in a sub-section of population $(\mathrm{N}=20)$ in Homa-Bay County. However, the data used for piloting was not included in the final analyses. Following piloting, the tools were adjusted, for clarity and consistency. Baseline survey was done to capture the acceptance of VMMC; circumcision status and uptake of HIV voluntary counseling and testing. In addition, factors affecting acceptability of VMMC and access to VCT were documented. This information was captured using structured and semi-structured questionnaires (reported in Ngetich et al., 2018).

\section{Intervention}

A dialogue was held to raise awareness of the benefits of VMMC for males as well as their female partners, including reducing the risk of HIV. The dialogue team was made up of a trained male VMMC promoter, a community advocate, and male VMMC provider and a series of the Community Health Workers that had participated in the baseline data collection. The information was gathered using dialogue guides and responses captured through note taking and taping. Sharing on factors and potential reasons for not accepting VMMC were documented.

\section{Community dialogue strategy}

The following areas were of VCT focus during the community dialogue:

i. perceptions regarding HIV and AIDS;

ii. knowledge about HIV Counselling and Testing (HCT);

iii. whether people living with HIV are stigmatized;

iv. basic infrastructure for HCT services delivery at community level;

v. care and support for people living with HIV and AIDs (PLWAs).

The following areas were of VMMC focus during the community dialogue:

i. Medical male circumcision is a one-time health intervention that provides men with life-long partial protection against HIV;

ii. Apart from reducing HIV infection, male circumcision decreases the risk of urinary tract infections; reduces risk of sexually transmitted diseases in men;

iii. protects against cancer of the penis

iv. prevents inflammation of the glands and foreskin 
v. Benefits of male circumcision to women: improving hygiene, reducing STI's, and reducing the risk of cervical cancer (Human Papilloma Virus - HPV).

vi. Discussion on results from several studies providing evidence that men who chose surgical VMMC were $60 \%$ less likely to be infected with HIV than men who were not circumcised.

The intervention was performed end of February 2018. The study allowed a period of 2 months (60 days) from March-April, 2018 and collected data on those who accessed VCT and VMMC services in Homa-Bay County following the community dialogue.

\section{End-Line Survey}

End-line survey was done to capture the acceptance of VMMC; circumcision status and uptake of HIV voluntary counseling and testing. This information was captured using structured and semistructured questionnaires.

\section{Data analysis}

Data analyses were performed using Statistical Package for Social Scientists (SPSS, version 22) software. Quantitative methods of data analysis were used. Descriptive statistics were used to analyze continuous variables. Chi-square test was used to establish proportionality. Changes in numbers accessing the services was used to determine the effect of dialogue on the uptake of voluntary medical male circumcision and HIV counseling and testing.

\section{Results}

\section{Demographic characteristics of the study participants}

A total of 419 respondents were drawn from three sub-counties within Homa-Bay County. The distributions of the 419 respondents were as follows: Ndhiwa Sub-county were 142 (33.9\%), Rachuonyo North were 138 (32.9\%) and Suba South were 139 (33.2\%). The data that follows are henceforth stratified as per these sub-counties. Table 1 presents the socio-demographic and socioeconomic characteristics of the study participants as stratified into these 3 sub-counties. In the three different sub-counties, majority were married i.e. $69.7 \%$ in Ndhiwa, $58 \%$ in Rachuonyo and $64.7 \%$ in Suba Sub-counties. The proportions of those under the different marital status $(P=0.041)$ were significantly different. Likewise, the proportions of those belonging to different religious groups $(P=0.001)$ were different since there was a higher proportion of those in Other Christians in Ndhiwa (38\%) and Rachuonyo (36.2\%) while Roman Catholic was highest in Suba South (42.4\%). Majority of the respondents were either farming or casual labourers across the different sub-counties and the proportions of those in different categories of professions were significantly different across the subcounties $(P<0.0001)$, with the monthly income $(P=0.004)$ also varying significantly across the 3 different counties. However, those resident in rural versus urban set-ups were comparable $(P=0.071)$. These might imply that the set-up in the different sub-counties in Homa-Bay County is generally different socio-demographically and socio-economically. We previously reported on this (Ngetich et al., 2018).

The effect of community dialogue on self-initiated voluntary medical male circumcision (VMMC) among male residents of Homa-Bay Country in Kenya.

In order to establish the effect of community dialogue on self-initiated voluntary medical male circumcision (VMMC) among male residents of Homa-Bay County in Kenya, data on those who accessed VMMC prior to the community dialogue and after the intervention were compared. Analysis showed that the numbers of males accessing VMMC services was generally increased from the time the community dialogue was performed. It moved from 829 (January, 2018), 1695 (February, 2018), 815 (March, 2018) and finally 3600 (April, 2018) (Figure 1). Collectively, the trend shows a general positive increment (from March to April) on the numbers of males accessing VMMC following community dialogue.

The effect of community dialogue on the uptake of HIV voluntary counseling and testing (VCT) among male residents of Homa-Bay County, Kenya.

To determine the effect of community dialogue on the uptake of HIV voluntary counseling and testing (VCT) among male residents of Homa-Bay County, Kenya, data on those who accessed VCT 
prior to the community dialogue and after the intervention were compared. The results revealed that just like for the VMMC, the numbers of males accessing VCT services was generally increased from the time the community dialogue was performed. It moved from 360 (January, 2018), 1281 (February, 2018), 615 (March, 2018) and finally 1729 (April, 2018). Consistently, the trend shows a general positive increment (from March to April) on the numbers of males accessing VCT following community dialogue (Figure 2).

\section{Discussion}

Community Dialogues has been used to address underlying social determinants, especially inequitable gender norms, as a fundamental step in reducing HIV risk behaviors, increasing selfefficacy to talk about and address HIV, and decreasing HIV stigma in countries such as Zimbabwe. However, in Kenya, it has been applied in several other areas such as in task shifting among community health workers in nomadic communities (Ochieng et al., 2014), in shifting social norms and enabling family planning initiatives (Wegs et al., 2016) and in identifying what motivates maternal and child nutrition peer educators based on fathers' and grandmothers' experiences in western Kenya (Martin et al., 2015). However, its usefulness in prevention of HIV has not previously been shown. This study therefore determined the effect of community dialogue on self-initiated voluntary medical male circumcision (VMMC) and uptake of HIV voluntary counseling and testing (VCT) among male residents of Homa-Bay County, Kenya.

Different countries have registered low uptake of VMMC services among the target populations. Some regions have registered much lower uptake levels than others especially among men 18 years old and above. There is very little literature available on utilization of VMMC, especially among the older men who are prime targets for the intervention in the priority countries (WHO, 2007). From 2005 to 2007, three randomized controlled clinical trials in Sub-Saharan Africa (SSA) demonstrated that male circumcision reduces the risk of female-to-male HIV transmission by about $60 \%$ (Ashengo et al., 2012). In March 2007, the WHO and the Joint United Nations Programme on HIV/AIDS (UNAIDS) recommended that voluntary medical male circumcision (VMMC) should be considered an important intervention for HIV prevention in settings with high HIV and low circumcision prevalence (WHO, 2007). Mathematical models have also estimated that VMMC scale-up across SSA could prevent up to 6 million new HIV infections and 3 million deaths by 2025 (Njeuhmeli et al., 2011). VMMC has been rolled out in 14 African countries starting in 2009, and WHO estimates that 11.7 million men have been circumcised as of December 2015 (WHO, 2015).

It has previously been shown that cultural and religious practices contribute to wide variation of circumcision prevalence (WHO, 2016). Several regions had close to universal prevalence, whereas 12 traditionally non-circumcising regions had lower circumcision prevalence, and constituted the Ministry of Health, Community Development, Gender, Elderly, and Children (MOHCDGEC) priority regions for VMMC (TACAIDS, . 2013) The national VMMC programme was launched by the MOHCDGEC following a situational analysis from 2007-2008 that reported high acceptability for VMMC among men and women in both traditionally circumcising and non-circumcising communities (Ashengo et al., 2012). The MOHCDGEC developed a VMMC Country Operational Plan targeting 2.2 million men aged 10-34 years with circumcision between 2014 and 2017 in the priority regions (Ashengo et al., 2012). Although the national strategy prioritized 10-34 years old, modeling suggested that the most immediate reduction to HIV incidence may be achieved by circumcising men aged 20-34 years because of higher HIV incidence in this age group (Ashengo et al., 2012). To achieve the maximum number of HIV infections averted (3\% of new infections) between 2011 and 2015, it was reported that VMMC should reach sexually active men (WHO, 2015). Studies carried out in Kenya, Zambia, and Tanzania have described strategies to increase VMMC demand among men aged 20 years or above (Kundi et al., 2013) but these strategies did not explicitly explore how a community dialogue approach can affect the uptake of VMMC services by men.

Following the community dialogue as an intervention, there was an upsurge of numbers of males accessing VMMC services in Homa-Bay County, pointing out to the fact that this was an effective intervention in this community. Kenya has integrated VMMC as part of the HIV prevention interventions. The overwhelming evidence of the effectiveness of VMMC in reducing the risk of 
acquiring HIV in men is compelling. However, while there has been so much progress on service delivery and strategic planning of the VMMC program in Kenya, there is also an increasing need to ensure that the general public is aware of VMMC and its benefits and risks through a comprehensive communication strategy, such as was demonstrated in the current study. In such approaches, it is critical to highlight the partial protection that VMMC offers and point out to choices and decisions that couples should make to make certain that VMMC does not increase the risk but rather reduces the risk of HIV infection.

The VMMC policy and the standard operating procedures have already set up the base for effective scale-up of the service and policy direction in African set-ups and beyond. It is therefore, imperative that an effective communication strategy aimed at improving the knowledge and understanding of VMMC within the general public is adopted to increase the demand for the service. VMMC is very effective if targets that have been set are achieved because of its public health benefit. As such, intense demand creation strategies are required while also acknowledging that all men that have been circumcised need to follow other safer sexual practices post-VMMC in order not to acquire or spread the disease. It is also important to promote VMMC for HIV prevention and delink it from the cultural and religious bases from which it has been associated with for a long time.

In this region, the current study demonstrated that there were more males accessing VCT than VMMC services. The study showed that since the males resident around the Lake Victoria traditionally has had higher risks of contracting HIV and AIDs, they made a deliberate attempt to highly access these services. Worldwide, many countries have recognized VCT services as a priority intervention for HIV/AIDS control (WHO, 2007). As much as there is adequate knowledge on the availability of VCT services in the current study population (and community) in accessing the VCT services, the methods of providing information to the population still need to be up-scaled. The use of community dialogue could be an additional resource to this upscaling. The approach, however, should be done with great care to avoid negative outcomes and ensure that it is fully accepted in the communities.

Lastly, VCT service should be closely monitored and evaluated with an aim of making improvements. According to the FHI (FHI, 2005), monitoring and evaluation is a critical component of VCT service delivery and should address two relevant areas for service providers and policy makers. For service providers, it should examine how well VCT service is provided while for policy makers, it should determine programme effectiveness, and the impact of VCT on the population receiving the service.

\section{Conclusion}

On the basis of the findings from this this study, use of community dialogue is an effective intervention and increases the number of males in accessing VMMC and VCT services following community dialogue. Therefore, these findings will advise interventions and help eliminate stigma associated with male circumcision hence promoting it as an intervention in prevention of HIV infection.

\section{Acknowledgments}

Much appreciation goes to the Maseno University Ethics Research Committee (MUERC) members for the approval and the Director of Health, Homa-Bay County, for his authority to carry out the study in Homa-Bay County. I extend my sincere thanks to one and all of the research team at Homa-Bay County and the study participants whose help was fundamental in the completion of this document.

$\begin{array}{ll}\text { Abbreviations } & \\ \text { AIDS } & \text { Acquired Immune Deficiency Syndrome } \\ \text { CBO } & \text { Community-Based Organizations } \\ \text { CI } & \text { Confidence Interval } \\ \text { FHI } & \text { Family Health International } \\ \text { HIV } & \text { Human Immunodeficiency Virus } \\ \text { KAIS } & \text { Kenya AIDS Indicator Survey }\end{array}$




$\begin{array}{ll}\text { KDHS } & \text { Kenya Demographic Health Survey } \\ \text { MDG } & \text { Millennium Development Goal } \\ \text { MOHCDGEC } & \text { Ministry of Health, Community Development, Gender, } \\ & \text { Elderly, and Children } \\ \text { MUERC } & \text { Maseno University Ethics Review Committee } \\ \text { NGO } & \text { Non-Governmental Organization } \\ \text { PEPFAR } & \text { President's Emergency Plan for AIDS Relief } \\ \text { SDG } & \text { Sustainable Development Goal } \\ \text { UNAIDS } & \text { The Joint United Nations Programme on HIV and } \\ \text { VCT } & \text { AIDS } \\ \text { VMMC } & \text { Voluntary Counseling and Testing } \\ \text { WHO } & \text { Voluntary Medical Male Circumcision }\end{array}$

\section{References}

[1]. Ashengo, T.A., Hatzold, K., Mahler, H., Rock, A., Kanagat, N., and Magalona, S. (2012). Voluntary medical male circumcision (VMMC) in Tanzania and Zimbabwe: service delivery intensity and modality and their influence on the age of clients. PLoS One 9, e83642.

[2]. Coates, T.J., Richter, L., and Caceres, C. (2008). Behavioural strategies to reduce HIV transmission: how to make them work better. Lancet 372, 669-684.

[3]. Exner, T.M., Hoffman, S., Parikh, K., Leu, C.S., and Ehrhardt, A. (2002). HIV counseling and testing: women's experiences and the perceived role of testing as a prevention strategy. Perspectives on sexual and reproductive health $34,76-83$.

[4]. FHI (2005). Family Health International (FHI). (2005). Service Delivery Models for HIV Counseling and Testing, FHI, Washington, DC, USA, 2005.

[5]. Gilbert, L., and Walker, L. (2010). 'My biggest fear was that people would reject me once they knew my status...': stigma as experienced by patients in an HIV/AIDS clinic in Johannesburg, South Africa. Health \& social care in the community $18,139-146$.

[6]. Govindasamy, D., Ford, N., and Kranzer, K. (2012). Risk factors, barriers and facilitators for linkage to antiretroviral therapy care: a systematic review. Aids 26, 2059-2067.

[7]. KAIS (2007). National AIDS and STI Control Program (NASCOP) Ministry of Health, Kenya) Kenya AIDS Indicator Survey; 2007.

[8]. KAIS (2012). National AIDS and STI Control Programme. Kenya AIDS Indicator Survey 2012: Final Report. Nairobi, Kenya: NASCOP, 2014.

[9]. KDHS (2008-2009). Kenya National Bureau of Statistics. Kenya Demographic and Health Survey. 20082009.

[10]. Kincaid, D.L. (1987). The convergence theory of communication, self-organization, and cultural evolution. In D. L. Kincaid (Ed.), Communication theory: Eastern and western perspectives New York, NY: Academic Press., 209-221.

[11]. Kincaid, D.L. (2004). From innovation to social norm: bounded normative influence. Journal of health communication 9 Suppl 1, 37-57.

[12]. Kundi, G.J., Mphuru, L., McMahan, J., Mwakipesile, P., Mwanasalli, S., and Simbeye, D. (2013). Increased uptake of voluntary medical male circumcision (VMMC) services among older men following mobile technology demand creation in Shinyanga and Simiyu, Tanzania. -ISDS 2013; 2013.

[13]. Martin, S.L., Muhomah, T., Thuita, F., Bingham, A., and Mukuria, A.G. (2015). What motivates maternal and child nutrition peer educators? Experiences of fathers and grandmothers in western Kenya. Social science \& medicine 143, 45-53.

[14]. Murdock, G.P. (1956). How culture changes. In H. L. Shapiro (Ed.), Man, culture, and society (pp. 247260). New York, NY: Oxford University Press.

[15]. Ngetich, A., Abon'go, B., Okomo, G., and Ouma, C. (2018). Community acceptability of voluntary medical male circumcision (VMMC) as a strategy in the fight against the spread of HIV and AIDS among residents of Homa-Bay County Kenya. Texila International Journal. 
[16]. Njeuhmeli, E., Forsythe, S., Reed, J., Opuni, M., Bollinger, L., Heard, N., Castor, D., Stover, J., Farley, T., Menon, V., and Hankins, C. (2011). Voluntary medical male circumcision: modeling the impact and cost of expanding male circumcision for HIV prevention in eastern and southern Africa. PLoS Med 8, e1001132.

[17]. Noelle-Neumann, E. (1974). The spiral of silence: A theory of public opinion. Journal of Communication 24, 43-51.

[18]. Noelle-Neumann, E. (2014). Public opinion and social control. In W. Donsbach, C. Salmon, \& Y. Tsfati (Eds.), The spiral of silence: New perspectives on communication and public opinion. New York, NY: Routledge., 19-32.

[19]. Ochieng, B., Akunja, E., Edwards, N., Mombo, D., Marende, L., and Kaseje, D.C. (2014). Perceptions of health stakeholders on task shifting and motivation of community health workers in different socio demographic contexts in Kenya (nomadic, peri-urban and rural agrarian). BMC health services research 14 Suppl 1, S4.

[20]. Sanjobo, N., Frich, J.C., and Fretheim, A. (2008). Barriers and facilitators to patients' adherence to antiretroviral treatment in Zambia: a qualitative study. SAHARA J: journal of Social Aspects of HIV/AIDS Research Alliance 5, 136-143.

[21]. Scheufele, D.A., and Moy, P. (2000). Twenty-five years of the spiral of silence: A conceptual review and empirical outlook. International Journal of Public Opinion Research 12, 3-28.

[22]. TACAIDS (. 2013). TACAIDS, ZAC, NBS, OCGS, International I. (2013). Tanzania HIV/AIDS and malaria indicator survey 2011-12: key findings. Dar es Salaam, Tanzania: National Bureau of Statistics, Ministry of Finance; 2013.

[23]. UNAIDS, W.a. (2016). A framework for voluntary medical male circumcision: VMMC 2021 [Internet]. 2016. [cited 2017 December 30th]. Available from: http://www.who.int/hiv/pub/malecircumcision.

[24]. Underwood, C., Brown, J., Sherard, D., and Abdur-Rahman, A. (2011). Reconstructing gender norms through ritual communication: A study of African Transformation. Journal of Communication 61, 197-218.

[25]. Wachira, J., Kimaiyo, S., Ndege, S., Mamlin, J., and Braitstein, P. (2012). What is the impact of homebased HIV counseling and testing on the clinical status of newly enrolled adults in a large HIV care program in Western Kenya? Clinical infectious diseases: an official publication of the Infectious Diseases Society of America 54, 275-281.

[26]. Wegs, C., Creanga, A.A., Galavotti, C., and Wamalwa, E. (2016). Community Dialogue to Shift Social Norms and Enable Family Planning: An Evaluation of the Family Planning Results Initiative in Kenya. PloS one 11, e0153907.

[27]. WHO (2007). WHO, UNAIDS. (2007). New data on male circumcision and HIV prevention (2007): policy and program implications. WHO/UNAIDS technical consultation on male circumcision and HIV prevention: research implications for policy and programming. WHO meeting Montreux, Switzerland. .

[28]. WHO (2015). WHO (2015). WHO progress brief voluntary medical male circumcision for HIV prevention in 14 priority countries in East and Southern Africa. Geneva, Switzerland: WHO; 2015.

[29]. WHO (2016). WHO. (2016). Voluntary Medical Male Circumcision for HIV prevention in 14 priority countries in east and southern africa. The 2016 WHO progress brief.

\section{Tables}

Table 1. Demographic characteristics of the study participants (adopted from Ngetich et al., 2018)

\begin{tabular}{|l|l|l|l|l|l|}
\hline & & $\begin{array}{l}\text { Ndhiwa Sub- } \\
\text { County }\end{array}$ & $\begin{array}{l}\text { Rachuonyo } \\
\text { North Sub- } \\
\text { County }\end{array}$ & $\begin{array}{l}\text { Suba South } \\
\text { Sub-County }\end{array}$ & $P$-value \\
\hline Marital status & Divorced & $2(1.4 \%)$ & $0(0.0 \%)$ & $1(0.7 \%)$ & $\mathbf{0 . 0 4 1}$ \\
\cline { 2 - 7 } & Married & $99(69.7 \%)$ & $80(58.0 \%)$ & $90(64.7 \%)$ & \\
\cline { 2 - 7 } & Separated & $2(1.4 \%)$ & $2(1.4 \%)$ & $1(0.7 \%)$ & \\
\hline
\end{tabular}


Texila International Journal of Public Health Volume 6, Issue 3, Sep 2018

\begin{tabular}{|c|c|c|c|c|c|}
\hline & Single & $32(22.5 \%)$ & $54(39.1 \%)$ & $46(33.1 \%)$ & \\
\hline & Widowed & $7(4.9 \%)$ & $2(1.4 \%)$ & $1(0.7 \%)$ & \\
\hline \multirow[t]{5}{*}{ Religion } & None & $12(8.5 \%)$ & $4(2.9 \%)$ & $3(2.2 \%)$ & 0.001 \\
\hline & Muslim & $3(2.1 \%)$ & $1(0.7 \%)$ & $4(2.9 \%)$ & \\
\hline & $\begin{array}{l}\text { Other } \\
\text { Christians }\end{array}$ & $54(38.0 \%)$ & $50(36.2 \%)$ & $33(23.7 \%)$ & \\
\hline & Protestant & $24(16.9 \%)$ & $47(34.1 \%)$ & $40(28.8 \%)$ & \\
\hline & $\begin{array}{l}\text { Roman } \\
\text { Catholic }\end{array}$ & $49(34.5 \%)$ & $36(26.1 \%)$ & $59(42.4 \%)$ & \\
\hline \multirow[t]{15}{*}{ Occupation } & Blacksmith & $0(0.0 \%)$ & $0(0.0 \%)$ & $1(0.7 \%)$ & $<0.0001$ \\
\hline & Jua Kali & $1(0.7 \%)$ & $1(0.7 \%)$ & $4(2.9 \%)$ & \\
\hline & $\begin{array}{l}\text { Not } \\
\text { Working }\end{array}$ & $17(12.0 \%)$ & $26(18.8 \%)$ & $24(17.3 \%)$ & \\
\hline & $\begin{array}{l}\text { Paid } \\
\text { Employment }\end{array}$ & $8(5.6 \%)$ & $13(9.4 \%)$ & $14(10.1 \%)$ & \\
\hline & Priests & $1(0.7 \%)$ & $0(0.0 \%)$ & $0(0.0 \%)$ & \\
\hline & Student & $1(0.7 \%)$ & $6(4.3 \%)$ & $2(1.4 \%)$ & \\
\hline & $\begin{array}{l}\text { Self- } \\
\text { Employed }\end{array}$ & $0(0.0 \%)$ & $0(0.0 \%)$ & $3(2.2 \%)$ & \\
\hline & Boda Boda & $2(1.4 \%)$ & $2(1.4 \%)$ & $0(0.0 \%)$ & \\
\hline & Carpentry & $0(0.0 \%)$ & $1(0.7 \%)$ & $0(0.0 \%)$ & \\
\hline & $\begin{array}{l}\text { Casual } \\
\text { Labourer }\end{array}$ & $34(23.9 \%)$ & $56(40.6 \%)$ & $34(24.5 \%)$ & \\
\hline & Contractor & $0(0.0 \%)$ & $0(0.0 \%)$ & $1(0.7 \%)$ & \\
\hline & Driver & $1(0.7 \%)$ & $0(0.0 \%)$ & $0(0.0 \%)$ & \\
\hline & Farming & $76(53.5 \%)$ & $26(18.8 \%)$ & $53(38.1 \%)$ & \\
\hline & Fishing & $0(0.0 \%)$ & $7(5.1 \%)$ & $3(2.2 \%)$ & \\
\hline & $\begin{array}{l}\text { Gold } \\
\text { Mining }\end{array}$ & $1(0.7 \%)$ & $0(0.0 \%)$ & $0(0.0 \%)$ & \\
\hline
\end{tabular}


DOI: $10.21522 /$ TIJPH.2013.06.03.Art005

ISSN: $2520-3134$

\begin{tabular}{|l|l|l|l|l|l|}
\hline $\begin{array}{l}\text { Monthly } \\
\text { Income }\end{array}$ & $\begin{array}{l}10,000- \\
20,000\end{array}$ & $32(22.5 \%)$ & $27(19.6 \%)$ & $22(15.8 \%)$ & $\mathbf{0 . 0 0 4}$ \\
\hline & $\begin{array}{l}>20,000- \\
50,000\end{array}$ & $6(4.2 \%)$ & $13(9.4 \%)$ & $5(3.6 \%)$ & \\
\hline & $<10,000$ & $95(66.9 \%)$ & $77(55.8 \%)$ & $101(72.7 \%)$ & \\
\hline & $>50,000$ & $4(2.8 \%)$ & $1(0.7 \%)$ & $2(1.4 \%)$ & \\
\hline & None & $5(3.5 \%)$ & $20(14.5 \%)$ & $9(6.5 \%)$ & \\
\hline Resident & Rural & $124(87.3 \%)$ & $129(93.5 \%)$ & $131(94.2 \%)$ & 0.071 \\
\hline & Urban & $18(12.7 \%)$ & $9(6.5 \%)$ & $8(5.8 \%)$ & \\
\hline
\end{tabular}

Analyses performed using Chi-square test. $P$-values in bold are significant at the $P \leq 0.05$ cut-off.

\section{Figures}

\section{Figure 1}

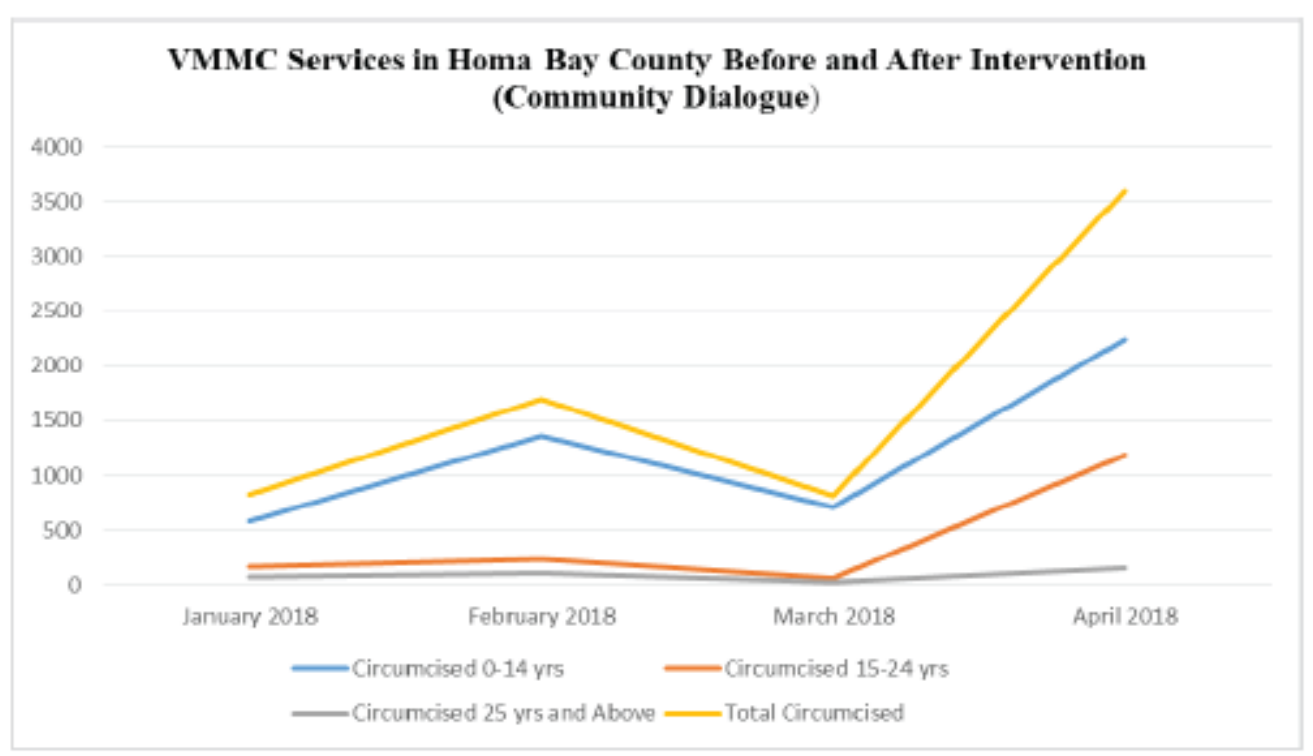

Figure 1. Effect of community dialogue on the number of those accessing VMMC services in Homa-Bay County. $\mathrm{X}$-axis are the months, $\mathrm{Y}$-axis are the numbers of males accessing VMMC services. 


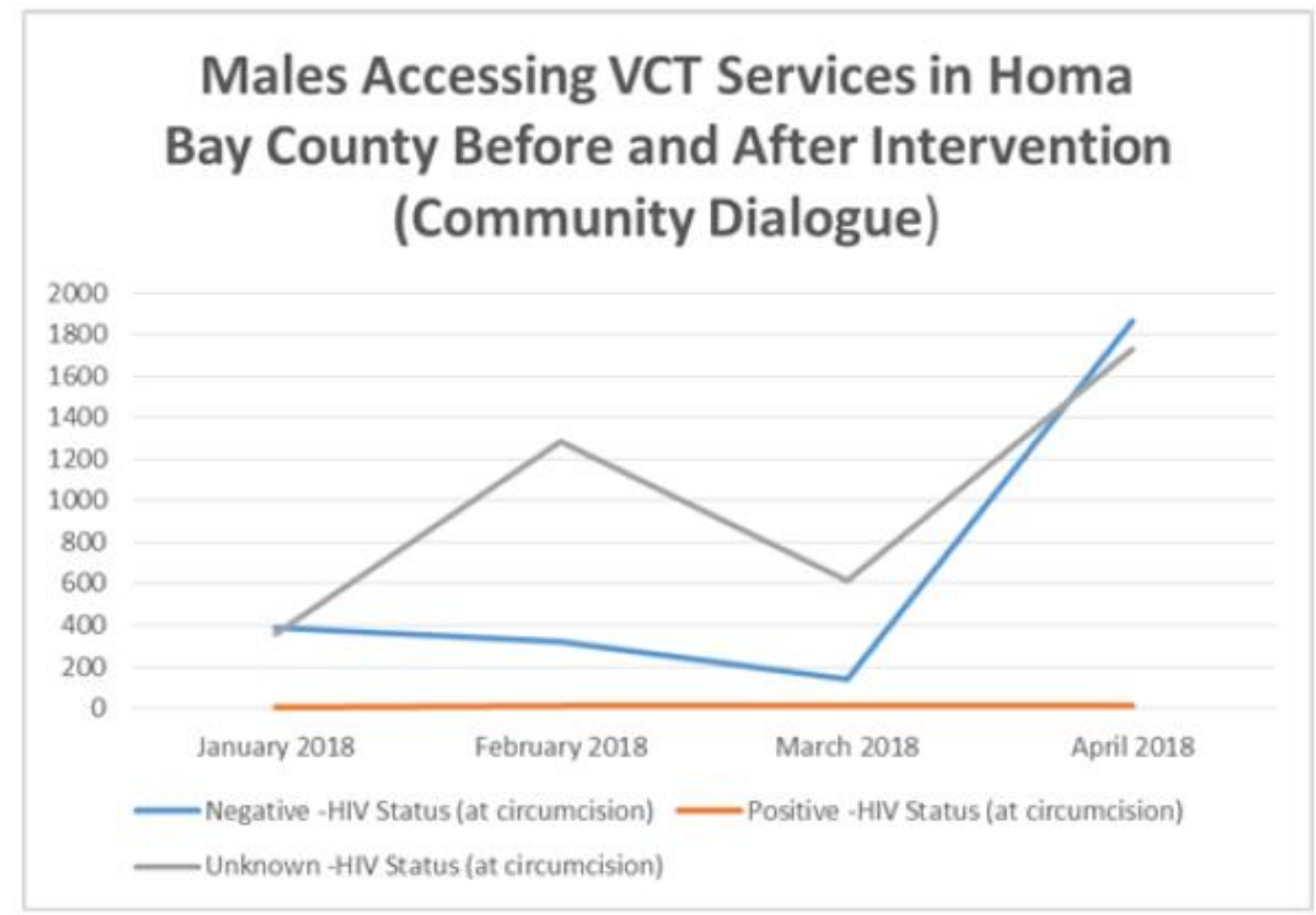

*The black line passing through =intervention.

Figure 2. Effect of community dialogue on the number of those accessing VCT services in Homa-Bay County. $\mathrm{X}$-axis are the months, Y-axis are the numbers of males accessing VCT services. 